\title{
Hadronic jet models today
}

\author{
Marek Sikora ${ }^{1}$ \\ ${ }^{1}$ Nicolaus Copernicus Astronomical Center, \\ Bartycka 18, 00-716 Warsaw, Poland \\ email: sikora@camk.edu.pl
}

\begin{abstract}
The matter content of relativistic jets in AGNs is dominated by a mixture of protons, electrons, and positrons. During dissipative events these particles tap a significant portion of the internal and/or kinetic energy of the jet and convert it into electromagnetic radiation. While leptons - even those with only mildly relativistic energies - can radiate efficiently, protons need to be accelerated up to energies exceeding $10^{16-19} \mathrm{eV}$ to dissipate radiatively a significant amount of energy via either trigerring pair cascades or direct synchrotron emission. Here I review various constraints imposed on the role of hadronic non-adiabatic cooling processes in shaping the high energy spectra of blazars. It will be argued that protons, despite being efficiently accelerated and presumably playing a crucial role in jet dynamics and dissipation of the jet kinetic energy to the internal energy of electrons and positrons, are more likely to remain radiatively passive in AGN jets.
\end{abstract}

Keywords. galaxies: active, (galaxies:) BL Lacertae objects: general, galaxies: jets, (galaxies:) quasars: general, gamma rays: theory

\section{Introduction}

Production of relativistic jets in AGN is very likely mediated by rotation of large scale magnetic fields in magnetosphere of black hole and/or accretion disk (Blandford 1976; Lovelace 1976; Phinney 1983; Camenzind 1986; McKinney \& Blandford 2009). This leads to a formation of Poynting flux dominated outflows. Those in turn can at some point be converted to the matter dominated jets (Komissarov et al. 2007; Tchekhovskoy et al. 2009; Lyubarsky 2010; Komissarov 2010), with a terminal Lorentz factor $\Gamma \sim P_{j} / \dot{M} c^{2}$ where $P_{j}$ is the rate of energy extraction from rotating $\mathrm{BH}$ and/or accretion disk and $\dot{M}$ is the mass flux. Depending on whether a jet is launched in the BH magnetosphere or by the accretion disk, the mass flux is expected to be dominated by electron/positron pairs or by protons.

Presence of protons in AGN jets is indicated by the low-energy cutoffs in the radio spectra of hot spots in radio-lobes (Blundell et al. 2006; Stawarz et al. 2007; Godfrey et al. 2009) and by circular polarization and Faraday Rotation in radio cores (Vitrishchak et al. 2008; Park \& Blackman 2010). If so, such protons might manifest their presence also via radiative contribution to high energy spectra in blazars, by synchrotron emission of pair cascades triggered by photo-meson process and by direct synchrotron emission of protons and mesons (Mannheim \& Biermann 1992; Mannheim 1993; Rachen \& Mészáros 1998; Aharonian 2000). Whether such a contribution can be significant is the main issue of this presentation. We start with a short review of the leading hadronic models $(\S 2)$; discuss efficiencies of the proton acceleration and cooling processes (§3); present observational constraints on hadronic contribution to blazar spectra ( $\$ 4)$. And, finally, we discuss the role of protons they play in the leptonic models regarding the aspect of preheating electrons up to thermal proton energies required to participate in further stochastic acceleration process $(\S 5)$. Main results are summarized in $\S 6$. 


\section{Hadronic models - basic features}

\subsection{Luminous blazars - 'photo-meson' models}

Luminous blazars are hosted by quasars which, when observed away from the axis of the jet, form population of FRII type double radio sources. They are powered by accretion onto $\mathrm{BHs}$ with masses typically of the order of $10^{9}$ solar masses and their accretion luminosities are in the range $10^{46}-10^{47} \mathrm{erg} \mathrm{s}^{-1}$. However, when such jets are oriented close to the line of sight, these quasars are seen as being dominated by nonthermal radiation of jets, with the apparent luminosities $10^{48-49} \mathrm{erg} \mathrm{s}^{-1}$ and spectra dominated by the broad high energy component peaking in the 1-100 $\mathrm{MeV}$ band. This radiation shows high amplitude variability on time scales from years down to days and even hours, implying the strong dissipative events taking place not far from the base of a jet. There the energy densities of the magnetic and radiation fields are very large, providing conditions for acceleration of protons up to energies of $10^{9-10} \mathrm{GeV}$, and for cooling them via inelastic collisions with soft photons (Sikora et al. 1987). For typical background radiation fields most of proton energy is converted to mesons and this initiates processes which according to proposers of hadronic models are responsible for $\gamma$-ray production in luminous blazars (Mannheim \& Biermann 1992).

These processes are dominated by the following channels. In approximately $90 \%$ collisions, the produced mesons are pions. In $2 / 3$ of them they are the neural pions $\left(\pi^{0}\right)$ and in $1 / 3$ of them - the positive pions $\pi^{+}$. Neutral pions almost immediately decay into photons which in turn trigger pair-cascades driven by photon-photon pair production and their synchrotron radiation. Escaping radiation is the product of 3rd and 4th generation of pairs (radiation of the first two are totally converted to $e^{+} e^{-}$-pairs). The resulting electromagnetic spectrum is predicted to form the high energy component peaked in the $\gamma$-ray band, with a high energy break at $h \nu_{b r} \sim 10-30 \mathrm{GeV}$, where $\tau_{\gamma \gamma \rightarrow e^{+} e^{-}} \simeq 1$, and low energy tail in the X-ray band with a slope $\alpha_{X}>0.5$.

Such spectra can be affected, but not significantly, by electromagnetic output resulting from production of charged pions via processes $p \gamma \rightarrow n+\pi^{+}$and $n \gamma \rightarrow p+\pi^{-}$. The charged pions decay producing muons $\left(\pi^{ \pm} \rightarrow \mu^{ \pm}+\bar{\nu}_{\mu} / \nu_{\mu}\right)$, and the muons decay producing positrons/electrons $\left(\mu^{ \pm} \rightarrow e^{ \pm}+\bar{\nu}_{e} / \nu_{e}+\nu_{\mu} / \bar{\nu}_{\mu}\right)$. The resulting electrons/positrons join the pair cascade triggered by the decays of the neutral pions and together with the synchrotron emission of muons (Rachen \& Mészáros 1998) contribute an additional $\sim 6-16 \%$ to the electromagnetic output of the photo-meson process.

\subsection{Low luminosity TeV BL Lac objects - proton-synchrotron models}

The low luminosity BL Lac objects are hosted by radio-galaxies of type FR I. Just as FR II radio sources, they are associated with giant elliptical galaxies, with central $\mathrm{BH}$ masses of the order of $10^{9} \mathrm{M}_{\odot}$, but with the jet powers at least 3 orders of magnitude lower than in the radio-loud quasars and extremely low accretion luminosities. Doppler boosted nonthermal radiation from their jets reaches $\mathrm{TeV}$ energies, and the luminosity peak of the high energy spectral component is located at $\mathrm{GeV}$ energies. Resulting from the low radiative environment, energy losses of ultra-relativistic protons in theses objects are presumably dominated by direct synchrotron emission of protons. This mechanism was suggested by Aharonian (2000) to be the primary source of $\gamma$-rays in low luminosity BL Lac objects. Main spectral features of such models, as is in the case of synchrotron radiation of electrons, are directly related to the magnetic field intensities and the injection function of relativistic particles (here of protons). They produce photons with average energies $\nu_{p, s y n}=\left(2 e /\left(3 \pi m_{p} c\right)\right) \gamma_{p}^{2} B^{\prime} \mathcal{D}$ and energy spectra with the slopes $\alpha=\left(q_{p}-1\right) / 2$ in the slow cooling regime and $\alpha=q_{p} / 2$ in the fast cooling regime, where $B^{\prime}$ is the intensity of magnetic field in the jet co-moving frame, $\mathcal{D}$ is the Doppler factor, $q_{p}$ is the 
index of the proton power-law injection function, $Q_{p} \propto \gamma_{p}^{-q_{p}}$, and $\alpha$ is the index of the radiation energy flux, $F_{\nu} \propto \nu^{-\alpha}$. Application of those formulae indicates that protons accelerated up to Lorentz factors $\gamma_{p} \sim 10^{10}$ and cooled in magnetic fields $B^{\prime} \sim 100$ Gauss may produce spectra reaching $\mathrm{TeV}$ energies and with the luminosity peak located near $\nu_{p, s y n, \max }$. An investigation whether these parameters are feasible - given the constraints imposed on $\gamma_{p, \max }$ by jets with the limited power and magnetisation - is given in $§ 4.2$.

\section{Radiative efficiencies}

\subsection{Time scales}

Acceleration.

Time scale of the proton acceleration as measured in the co-moving frame of the flow is

$$
t_{a c c}^{\prime}=f R_{L} / c=\frac{m_{p} c}{e} \frac{f \gamma_{p}}{B^{\prime}},
$$

where $R_{L}$ is the Larmor radius, $B^{\prime}$ is the magnetic field intensity, and $f$ is the parameter which in the case of shock acceleration depends on the spectrum of magnetic turbulence and on the velocity of the upstream-flow (Rieger et al. 2007) and for mildly relativistic shocks is expected to be at least of the order of 10 .

\section{Adiabatic losses}

Assuming that cross-sectional radius of the source is of the order of the cross-sectional radius of the jet, $R$, relativistic plasma moving down the jet with a Lorentz factor $\Gamma$ undergoes energy losses due to adiabatic expansion. For conical jets with the half-opening angle $\theta_{j} \equiv R / r<1 / \Gamma$, where $r$ is the distance of the source in a jet, the time scale of the adiabatic losses is (Moderski et al. 2003)

$$
t_{a d}^{\prime}=\frac{3}{2} \frac{R}{\left(\theta_{j} \Gamma\right) c} .
$$

\section{Photo-meson process}

Time scale of the energy losses via the photo-meson process can be estimated using the approximate formula (Begelman et al. 1990)

$$
t_{p \gamma}^{\prime} \sim \frac{1}{\left\langle\sigma_{p \gamma} K_{p \gamma}\right\rangle c n_{p h}^{\prime}\left(\nu^{\prime}>\nu_{t h}^{\prime}\right)}
$$

where $\left\langle\sigma_{p \gamma} K_{p \gamma}\right\rangle \sim 0.7 \times 10^{-28} \mathrm{~cm}^{2}$ is the product of the photo-meson cross-section and inelasticity parameter averaged over the resonant energy range, $n_{p h}^{\prime}\left(\nu^{\prime}>\nu_{t h}^{\prime}\right)=$ $\int_{\nu_{t h}^{\prime}} n_{\nu}^{\prime} \mathrm{d} n_{\nu}^{\prime}, h \nu_{t h}^{\prime} \simeq m_{\pi} c^{2} / \gamma_{p}$ is the threshold photon energy and $m_{\pi}$ is the rest mass of the pion. The target radiation field is provided by the internal and external sources. The internal seed soft radiation is dominated by synchrotron emission of primary (directly accelerated) electrons, the external one - by re-scattered/reprocessed disk radiation. Approximating the broad synchrotron spectral component by a power-law function with the energy-flux index $\alpha=1$ and denoting its luminosity by $L_{s}$, we have

$$
n_{p h(i n t)}^{\prime}\left(\nu^{\prime}>\nu_{t h}^{\prime}\right) \sim \frac{L_{s}}{4 \pi m_{\pi} c^{3} R^{2} \mathcal{D}^{4}} \gamma_{p} .
$$

Spectra of external radiation fields are in turn narrow and therefore can be approximated by mono-energetic functions. Hence,

$$
n_{p h(e x t)}^{\prime} \sim \frac{\xi L_{d} \Gamma}{4 \pi c r^{2} h \nu_{e x t}}=\frac{\xi L_{d}\left(\theta_{j} \Gamma\right)^{2}}{4 \pi c R^{2} \Gamma h \nu_{e x t}} \text { for } \gamma_{p}>m_{\pi} c^{2} / h \nu_{e x t},
$$


and $n_{p h, e x t}^{\prime}=0$ for $\gamma_{p}<m_{\pi} c^{2} /\left(\Gamma h \nu_{e x t}\right)$, where $L_{d}$ is the luminosity of the accretion disk and $\xi$ is the fraction of this luminosity rescattered/reprocesssed on a spatial scale corresponding with a distance $r$ of the source in a jet.

\section{Synchrotron emission}

Time scale of the proton cooling via the synchrotron process is

$$
t_{p, s y n}^{\prime}=\frac{3}{4}\left(\frac{m_{p}}{m_{e}}\right)^{3} \frac{m_{e} c}{\sigma_{T} u_{B}^{\prime}} \frac{1}{\gamma_{p}},
$$

where $u_{B}^{\prime}=B^{\prime 2} /(8 \pi)$ is the magnetic energy density.

\section{2. 'Mono-energetic' efficiencies}

In order to illustrate efficiencies of the proton acceleration and cooling processes we introduce their dimensionless rates, as scaled by the adiabatic losses rates, $\tau_{i} \equiv t_{i}^{\prime-1} / t^{\prime}{ }_{a d}$. They are:

$$
\begin{gathered}
\tau_{a c c} \simeq 4.8 \times 10^{-7} \frac{B^{\prime} R}{f\left(\theta_{j} \Gamma\right) \gamma_{p}} \simeq 0.8 \frac{(\sigma / 0.1)^{1 / 2} L_{j, 47}^{1 / 2}}{(f / 10)(\Gamma / 10)\left(\theta_{j} \Gamma\right)} \frac{1}{\gamma_{p, 10}}, \\
\tau_{p \gamma}^{(i n t)} \sim 1.2 \times 10^{-36} \frac{L_{s} \gamma_{p}}{\left(\theta_{j} \Gamma\right) \mathcal{D}^{4} R} \simeq 12 \frac{L_{s, 47}}{\left(\theta_{j} \Gamma\right)(\Gamma / 10)^{4} R_{16}} \gamma_{p, 10}, \\
\tau_{p \gamma}^{(e x t)} \sim 2.8 \times 10^{-40} \frac{\xi L_{d}\left(\theta_{j} \Gamma\right)}{h \nu_{e x t} \Gamma R} \simeq 0.2 \frac{\left(\xi L_{d}\right)_{45}\left(\theta_{j} \Gamma\right)}{\left(h \nu_{e x t} / 10 \mathrm{eV}\right)(\Gamma / 10) R_{16}},
\end{gathered}
$$

and

$$
\tau_{p, s y n}=1.1 \times 10^{-29} \frac{R B^{\prime 2} \gamma_{p}}{\theta_{j} \Gamma} \simeq 0.3 \frac{(\sigma / 0.1) L_{j, 47}}{\left(\theta_{j} \Gamma\right)(\Gamma / 10)^{2} R_{16}} \gamma_{p, 10},
$$

where $L_{B} \simeq c u_{B}^{\prime} \pi R^{2} \Gamma^{2}=\sigma L_{j} /(1+\sigma)$ is the magnetic energy flux, $L_{j}=L_{M}+L_{B}$ is the total jet power, $\sigma \equiv L_{B} / L_{M}$, and $L_{M}$ is the energy flux of the rest mass. Assuming $\sigma<1$ we use approximation $L_{B} \sim \sigma L_{j}$.

The 'scaled' quantities, $L_{j, 47}, \gamma_{p, 10}, R_{16}, L_{s, 47}$, and $\left(\xi L_{d}\right)_{46}$ are defined in the usual way, i.e. $X_{n} \equiv X / 10^{n}$.

\subsection{Maximal proton energies}

Maximal proton energies - if limited only by adiabatic losses - can be found from $\tau_{a c c}=1$ to be

$$
\gamma_{p}\left(\tau_{a c c}=1\right) \simeq 8 \times 10^{9} \frac{(\sigma / 0.1)^{1 / 2} L_{j, 47}^{1 / 2}}{(f / 10)(\Gamma / 10)\left(\theta_{j} \Gamma\right)},
$$

Stronger limits are imposed if dominant energy losses are non-adiabatic, i.e. for $\tau_{\text {cool }}=$ $\tau_{p \gamma}+\tau_{p, s y n}>1$. For energy losses dominated by photo-meson process with the target radiation field provided by internal sources or by proton-synchrotron radiation

$$
\gamma_{p, \max }=\gamma_{p}\left(\tau_{a c c}=1\right) \operatorname{Min}\left[1 ; \sqrt{R / R_{c}}\right] .
$$

where in the 1st case (photo-meson process)

$$
R_{c}^{(p \gamma)} \simeq 9.5 \times 10^{16} \frac{L_{s, 47}(\sigma / 0.1)^{1 / 2} L_{j, 47}^{1 / 2}}{\left(\theta_{j} \Gamma\right)(\Gamma / 10)^{5}(f / 10)}[\mathrm{cm}] .
$$

and in the 2nd case (proton-synchrotron radiation)

$$
R_{c}^{(s y n)} \simeq 2.3 \times 10^{15} \frac{(\sigma / 0.1)^{3 / 2} L_{j, 47}^{3 / 2}}{(f / 10)\left(\theta_{j} \Gamma\right)(\Gamma / 10)^{3}}[\mathrm{~cm}] .
$$


For energy losses dominated by photo-meson process with the target radiation field provided by external sources, $\tau_{p \gamma}^{(e x t)}$ is predicted to be lower than unity for any proton energy and therefore $\gamma_{p, \max }$ is not expected to be affected.

\subsection{Total efficiencies}

Radiative efficiency of a given cooling process can be estimated using formula

$$
\eta_{i} \simeq \frac{\int_{1}^{\gamma_{p, \text { max }}} \eta_{i}\left(\gamma_{p}\right) Q_{\gamma_{p}} \gamma_{p} d \gamma_{p}}{\int_{1}^{\gamma_{p, \text { max }}} Q_{\gamma_{p}} \gamma_{p} d \gamma_{p}}
$$

where $\eta_{i}\left(\gamma_{p}\right) \simeq \operatorname{Min}\left[\tau_{i} ; 1\right]$ and $Q_{\gamma_{p}}$ is the proton injection function.

We calculate such efficiencies below assuming power-law injection of protons $Q_{\gamma_{p}} \propto$ $\gamma_{p}^{-q_{p}}$ with $q_{p}=2$. This specific value of the index is chosen because efficiencies obtained for $q_{p}=2$ provide upper limits of efficiencies available for $q_{p}>2$ being predicted by theoretical models of a diffusive acceleration of particles in mildly relativistic shocks and supported by the slopes of synchrotron spectra produced in the IR-Optical bands by primary (directly) accelerated electrons/positrons.

For energy losses dominated by photo-meson process with internally produced seed photons and by proton-synchrotron emission, we obtain

$$
\eta_{i} \simeq \frac{1+\ln \left(\gamma_{p, \max } / \gamma_{p 1}\right)}{\ln \gamma_{p, \max }} \text { for } R<R_{c}
$$

and $\eta_{i}<1 / \ln \gamma_{p, \text { max }}$ for $R>R_{c}$, where in case of 'internal' photo-meson process, $R_{c}$ is given by Eq.(13) and

$$
\gamma_{p 1}^{(p \gamma)}=\gamma_{p}\left(\tau_{p \gamma}^{(i n t)}=1\right) \simeq 8.3 \times 10^{8} \frac{\left.\left(\theta_{j} \Gamma\right)\right)(\Gamma / 10)^{4} R_{16}}{L_{s, 47}},
$$

while in case of proton-synchrotron emission, $R_{c}$ is given by Eq.(14) and

$$
\gamma_{p 1}^{(\text {syn })}=\gamma_{p}\left(\tau_{p, s y n}\right) \simeq 3.4 \times 10^{10} \frac{\left(\theta_{j} \Gamma\right)(\Gamma / 10)^{2} R_{16}}{(\sigma / 0.1) L_{j, 47}}
$$

For energy losses dominated by photo-meson process with externally produced seed photons, at any distance larger than

$$
r=\frac{\Gamma R\left(\tau_{p, \gamma}^{(e x t)}=1\right)}{\left(\theta_{j} \Gamma\right)} \simeq 1.8 \times 10^{16} \frac{\left(h \nu_{e x t} / 10 \mathrm{eV}\right)(\Gamma / 10)^{2}}{\left(\xi L_{d}\right)_{45}\left(\theta_{j} \Gamma\right)^{2}}[\mathrm{~cm}]
$$

$\tau_{p, \gamma}^{(e x t)} \leqslant 1$ and the efficiency is

$$
\eta_{p \gamma}^{(e x t)} \simeq \tau_{p \gamma}^{(e x t)} \frac{\ln \left(\gamma_{p, \max } / \gamma_{p, t h}\right)}{\ln \gamma_{p, \max }}
$$

where $\gamma_{p, t h}=m_{\pi} c^{2} /\left(\Gamma h \nu_{e x t}\right) \simeq 1.4 \times 10^{6} /\left((\Gamma / 10)\left(h \nu_{\text {ext }} / 10 \mathrm{eV}\right)\right)$.

\section{Observational constraints}

\subsection{Luminous blazars}

In order to account for the $\gamma$-ray luminosities of powerful blazars, radiative efficiency has to be

$$
\eta_{i}>0.3 \frac{L_{\gamma, 48}}{(\Gamma / 10)^{2}\left(\eta_{\text {diss }} / 0.3\right) L_{j, 47}}
$$


where $\eta_{\text {diss }}$ is the fraction of the jet energy flux dissipated in the 'blazar zone'. As it can be verified using approximate formulae presented in $\S 3.4$, such efficiency is difficult to reach even for protons injected with the energy distribution slopes $q_{p}=2$. For our fiducial parameters it is about $10 \%$ for the photo-meson process with intenally produced seed photons and much less for others. For slope $q_{p} \sim 2.4$ the efficiency drops to $\eta \sim 10^{-3}$.

Hadronic models may have also problems to explain very hard X-ray spectra of luminous blazars. Those blazars often have slopes $\alpha_{x}<0.5$ (see Table 1 in Sikora et al. 2009 ) and in order to explain such spectra by synchrotron radiation of secondary $e^{ \pm}-$ products of the cascades powered by hadrons - one needs to assume inefficient cooling of ultra-relativistic electrons/positrons up to energies

$$
\gamma_{e}>8.0 \times 10^{5} \frac{(h \nu / 100 \mathrm{keV})^{1 / 2}}{B^{\prime}(\Gamma / 10)} .
$$

Inefficient cooling of such energetic electrons/positrons implies very weak magnetic fields and, therefore, puts strong constraints on the efficiency of the proton acceleration and on efficiency of photo-meson energy losses via limitation of the maximal proton energy. Furthermore, as Sikora et al. (2009) demonstrated, in order to avoid overproduction of $\mathrm{X}$-rays in these magnetically weak sources by SSC radiation of primary electrons, it is necessary to assume the source sizes of the order of parsecs, and for such sources the efficiency of the photo-meson process is further reduced.

\subsection{Low luminosity BL Lac objects}

In these objects, because of low radiation energy densities - both in jets themselves and in the surroundings of the jet - the non-adiabatic energy losses of protons are presumably dominated by the proton-synchrotron mechanism. However, noting that such objects are hosted by weak radio-galaxies, with the jet powers $L_{j} \leqslant 10^{44} \mathrm{erg} \mathrm{s}^{-1}$, efficiency of synchrotron-proton models is also expected to be strongly reduced because weaker magnetic fields. In order to keep them at reasonable level more compact sources must be assumed. However, even in the case of most relativistic protons, the required size of the source to provide sufficiently strong magnetic fields for efficient cooling is unreasonably small (see Eq. (3.14),

$$
R_{c} \sim 0.7 \times 10^{11} \frac{(\sigma / 0.1)^{3 / 2} L_{j, 44}^{3 / 2}}{(f / 10)\left(\theta_{j} \Gamma\right)(\Gamma / 10)^{3}}[\mathrm{~cm}] .
$$

This is 3 orders less than the gravitational radius of the $\mathrm{BH}$ with mass $M_{B H} \sim 10^{9} M_{\odot}$. Considering the minimal cross-sectional radius of a jet to be $R \sim 10^{15} \mathrm{~cm}$, which for $\theta_{j} \sim 1 / \Gamma$ corresponds with a distance 100 gravitational radii of the $10^{9} M_{\odot} \mathrm{BH}-$ required to be at least of this order to accelerate the jet up to $\Gamma \sim 10$ (Komissarov et al. 2007) we can find using Eqs. (3.10) and (3.11) that

$$
\tau_{p, s y n}\left(\gamma_{p, \text { max }}\right) \simeq 0.7 \times 10^{-4} \frac{\left.(\sigma / 0.1) L_{j, 44}\right)^{3 / 2}}{(f / 1)\left(\theta_{j} \Gamma\right)(\Gamma / 10)^{3} R_{15}} .
$$

This indicates that even for such extreme parameters as $f \sim 1$ and $\sigma \sim 1$ the efficiency is too small to explain $\gamma$-ray luminosities $L_{\gamma} \sim 10^{44} \mathrm{erg} \mathrm{s}^{-1}$, unless one assumes very hard $\left(q_{p}<1\right)$ proton injection spectra and adopts significantly larger total jet power.

The main purpose of proton-synchrotron models was to explain the relatively stable shape of the TeV spectra in variable low luminosity BL Lac objects (Aharonian 2000). Obviously, the critical issue of such models is whether protons can reach sufficiently large energies to produce synchrotron spectra extending up to $\mathrm{TeV}$ energies. Combining 
formulae for average synchrotron photon energies, magnetic energy flux, and maximal proton energies (Eq. 3.11) gives

$$
h \nu_{p, \text { syn }, \text { max }} \simeq 3 \times 10^{-5} \frac{(\sigma / 0.1)^{3 / 2} L_{j, 44}^{3 / 2}}{(f / 10)^{2}(\Gamma / 10)^{2}\left(\theta_{j} \Gamma\right)^{2} R_{15}}[\mathrm{TeV}] .
$$

One can see that spectra may extend to TeV energies only if assuming $f \sim 1, \sigma \sim 1$, and jet powers $L_{j}>10^{45} \mathrm{erg} \mathrm{s}^{-1}$.

\section{Protons in leptonic models}

Disproving interpretation of high energy spectra of blazars produced via hadronic models does not disprove the presence of protons in AGN jets. They simply are expected to be radiatively inefficient but are likely to dominate the jet energy flux and strongly affect the dynamics of dissipation processes via shocks in the regions of low magnetization parameter $(\sigma<0.1)$. In this scenario, a large fraction of dissipated energy must be converted to relativistic electrons, otherwise leptonic models will be inefficient despite high radiative efficiencies of energetic electrons. However in order to get electrons to participate together with protons in the diffusive shock acceleration process, electrons must be first preheated up to average energy (to be strict - up to average momentum) of protons heated by randomization and compression in the shocked plasma. Several scenarios have been investigated for such electron preheating, both analytically and in PIC-simulations (Amato \& Arons 2006; Amano \& Hoshino 2009; Sironi \& Spitkovsky 2010). Some of them indicate the formation of a power-law energy distribution with the slope $1<q_{e}<2$. If it is true, then the number of electrons joining protons in the stochastic acceleration process can be lower than the number of protons by significant factor. Hence, the high efficiency of blazar radiation may indicate that $q_{e}<1$, or that there is a significant pair content.

In the ERC (External-Radiation-Compton) models (Sikora et al. 1994) of $\gamma$-ray production in luminous blazars such a low-energy tail should be observed in the $30 \mathrm{keV}-1$ $\mathrm{MeV}$ spectral energy range. Unfortunately, these bands are observationally poorly covered, particularly above $20 \mathrm{keV}$. A number of blazars have been detected up to $\sim 50 \mathrm{keV}$, by INTEGRAL (Beckmann et al. 2009), by Swift/BAT (Ghisellini et al. 2010), and by others (see Table 1 in Sikora et al. 2007). Some of them have spectral slopes corresponding with $1<q_{e}<2$. This, together with the location of the break in the energy range $1-10 \mathrm{MeV}$ as suggested by CGRO/COMPTEL and OSSE observations (Zhang et al. 2005; McNaron-Brown et al. 1995), may indicate a moderate $\left(n_{e} / n_{p} \sim 10\right)$ pair content, implied also by studies of the bulk-Compton and Compton-rocket effects (Sikora \& Madejski 2000; Ghisellini \& Tavecchio 2010). At energies $<20 \mathrm{keV}$ most blazars have softer $\mathrm{X}$-ray spectra, but they can result from the contribution of the SSC process and/or from the superposition of X-rays produced at different locations in a jet, such as the orphan X-ray outburst detected in 3C279 (Abdo et al. 2010).

\section{Conclusions}

For realistic jet powers $\left(L_{j} \leqslant L_{E d d}\right.$ in luminous blazars and $L_{j} \leqslant 10^{-3} L_{E d d}$ in low luminosity BL Lac objects), limited magnetization $(\sigma \leqslant 0.1$ is required to allow formation of strong shocks), and moderately steep proton injection function $\left(q_{p} \geqslant 2\right.$ is suggested by IR-optical spectra), the hadronic models fail to:

- reproduce $\gamma$-ray luminosities of blazars; 
- explain formation of very hard X-ray spectra in luminous blazars;

- provide the spectral extension up to TeV energies in low luminosity blazars.

Nevertheless, as indicated by several independent observations, protons are present in AGN jets and presumably play key role in dissipation processes in shocks. In particular, they transfer a fraction of the dissipated energy to electrons/positrons helping them to reach threshold energies for further acceleration by stochastic mechanisms and produce the observed $\gamma$-ray spectra via the ERC and SSC scenarios. However, significant pair content may be required to achieve reasonable effiecincy of that energy transfer.

\section{Acknowledgements}

I thank G. Madejski, K. Nalewajko and E. Stawarz for helpful comments. The work was supported by Polish grant MNiSW NN203 301635.

\section{References}

Abdo, A. A., et al. 2010, Nature, 463, 919

Aharonian, F. A. 2000, New Astron., 5, 377

Amato, E. \& Arons, J. 2006, ApJ, 653, 325

Amano, T. \& Hoshino, M. 2009, ApJ, 690, 244

Beckmann, V., Ricci, C., \& Soldi, S. 2010, arXiv: 0912.2254

Begelman, M. C., Rudak, B., \& Sikora, M. 1990, ApJ, 362, 38

Blandford, R. D. 1976, MNRAS, 176, 465

Blundell, K. M., Fabian, A. C., Crawford, C. S., et al. 2006, ApJ, 644, L13

Camenzind, M. 1986, A\&A, 156, 137

Ghisellini, G., Della Ceca, R., Volonteri, M., et al. 2010, MNRAS, 405, 387

Ghisellini, G. \& Tavecchio, F. 2010, arXiv: 1008.1982

Godfrey, L. E. H., Bicknell, G. V., Lovell, J. E. J., et al. 2009, ApJ, 695, 707

Komissarov, S. S. 2010, arXiv: 1006.2242

Komissarov, S. S., Barkov, M. V., Vlahakis, N., \& Königl, A. 2007, MNRAS, 380, 51

Lovelace, R. V. E. 1976, Nature, 262, 649

Lyubrasky, Y. E. 2010, MNRAS, 402, 353

Mannheim 1993 1993, A\&A, 269, 67

Mannheim, K. \& Biermann. P. L. 1992, A\&A, 253, L21

McKinney, J. C. \& Blandford, R. D. 2009, MNRAS, 394, L126

McNaron-Brown, K., Johnson, W. N., Jung, G. V., et al. 1995, Apj, 451, 575

Moderski, R., Sikora, M., \& Błażejowski, M. 2003, A\&̇A, 406, 855

Park, K. \& Blackman, E. G. 2010, MNRAS, 403, 1993

Phinney, E. E. 1983, PhD Thesis, Cambridge University

Rachen, J. P. \& Mészáros, P. 1998, Phys.Rev.D, 58, 123005

Rieger, F. M., Bosch-Ramon, V., \& Duffy, P. 2007, ApSS, 309, 119

Sikora, M., Begelman, M. C., \& Rees, M. J. 1994, ApJ, 421, 153

Sikora, M., Kirk, J., Begelman, M., \& Schneider, P. 1987, ApJ, 320, L81

Sikora, M. \& Madejski 2000, ApJ, 534, 109

Sikora, M., Stawarz, Ł., Moderski, M., Nalewajko, K., \& Madejski, G. M. 2009, ApJ, 704, 38

Sironi, L. \& Spitkovsky, A. 2010, arXiv: 1009.0024

Stawarz, Ł., Cheung, C. C., Harris, D. E., \& Ostrowski, M. 2007, ApJ, 662, 213

Tchekhovskoy, A., McKinney, J. C., \& Narayan, R. 2009, ApJ, 699, 1789

Vitrishchak, V. M., Gabuzda, D. C., Algaba, et al. 2008, MNRAS, 391, 124

Zhang, S., Collmar, W., \& Schönfelder, V. 2005 A\&A, 444, 767

\section{Discussion}


BEDNAREK: Can curvature energy losses of protons be important in AGNs?

SIKORA: They cannot be. This is because following particle acceleration processes in shocks or reconnection regions, relativistic protons are injected with a broad distribution of pitch angles. Such protons (as well as all other charged particles) spiral around magnetic field lines rather than slide on them.

YUAN: There are two kinds of B field in a jet. One is a large scale helical field, another being turbulent in a shock front. When we calculate radiation spectrum, we use turbulent one, but when we calculate polarization, we seem to use the large scale ordered field.

SIKORA: Properly calculated polarization must take into account both, the tangled/turbulent magnetic fields compressed and/or generated in a shock, as well, as the large scale magnetic fields transmitted through the shock.

DERMER: Acceleration to high energies is faster for ions than protons. How do your conclusions change if you consider Fe rather than p?

SIKORA: For approximately solar abundances of AGN plasmas the number of heavy nuclei is $\gg Z$ times smaller than of protons. Therefore, despite the fact that heavy nuclei are accelerated $Z$ times faster, they contribute to radiative processes much less than protons and our main conclusion that hadronic models cannot reproduce $\gamma$-ray luminosities of blazars remains valid.

PIRAN: Auger indicates that UHECRs are nuclei. It is much easier to accelerate nuclei. Nuclei will diffuse in the intergalactic field and can propagate only up to $\sim 10 \mathrm{Mpc}$. This suggestes that Cen A is the main source of UHECRs if those are nuclei and if the intergalactic magnetic field is $>10^{-9} \mathrm{G}$ (Piran et al. 2010).

SIKORA: Yes, I agree that Cen A may contribute significantly to the observed UHECRs. 